\title{
Overexpression of SCARA5 inhibits tumor proliferation and invasion in osteosarcoma via suppression of the FAK signaling pathway
}

\author{
XINZHU WEN ${ }^{1}$, NAN WANG $^{1}$, FANFAN ZHANG $^{2}$ and CHUNJIAO DONG ${ }^{3}$ \\ Departments of ${ }^{1}$ Orthopedics and ${ }^{2}$ Peripheral Vascular Surgery, \\ Dongfang Hospital Affiliated to Beijing University of Chinese Medicine; ${ }^{3}$ Department of Pneumology, \\ Dongzhimen Hospital Eastern Affiliated to Beijing University of Chinese Medicine, Beijing 100078, P.R. China
}

Received March 23, 2015; Accepted December 23, 2015

DOI: $10.3892 / \mathrm{mmr} .2016 .4857$

\begin{abstract}
Scavenger receptor class A, member 5 (SCARA5) is a member of the scavenger receptor family, and is involved in several types of human malignancy; however, its roles in osteosarcoma (OS) remain to be fully elucidated. Therefore, in the present study, the biological functions of SCARA5 in OS, and the potential underlying mechanisms were investigated. SCARA5 expression in OS tissues and cell lines was detected by reverse transcription-quantitative polymerase chain reaction and western blot analysis. The effects of SCARA5 on the proliferation and migration/invasion ability of OS cells were determined by MTT and Transwell chamber assays, respectively. Expression levels of phosphorylated focal adhesion kinase (p-FAK), FAK, p-Src, Src, matrix metalloproteinase (MMP)2 and MMP9 were evaluated via western blot analysis. The results of the present study demonstrated that SCARA5 was expressed at low levels in OS tissues and cell lines. The overexpression of SCARA5 significantly inhibited the proliferation, colony formation and migration/invasion abilities of the OS cells. Furthermore, SCARA5 significantly decreased the expression levels of p-FAK, MMP-2 and MMP-9 in the OS cells. Taken together, these data suggested that the overexpression of SCARA5 inhibits tumor proliferation and invasion in OS via suppression of the FAK signaling pathway. Thus, novel therapeutic strategies or drugs targeted at SCARA5 may offer potential for the treatment of OS.
\end{abstract}

\section{Introduction}

Osteosarcoma (OS) is the most prevalent primary malignant bone tumor, which predominantly affects children

Correspondence to: Dr Nan Wang, Department of Orthopedics, Dongfang Hospital Affiliated to Beijing University of Chinese Medicine, 6, Block 1, Fangxingyuan Fangzhuang, Fengtai, Beijing 100078, P.R. China

E-mail: wang_nan010@126.com

Key words: scavenger receptor class A, member 5, osteosarcoma, invasion, focal adhesion kinase signaling pathway and adolescents (1). Over the past decade, the development of multiple therapeutic strategies for OS has significantly enhanced patient outcomes, and the 5-year survival rate of patients with OS has improved markedly (2). However, outcomes remain poor, and the majority of patients eventually succumb to pulmonary metastases-associated mortality (3). Therefore, there is an urgent requirement to identify biomarkers and therapeutic targets for the treatment of patients with OS.

Class A scavenger receptors (SR-As) are type of cell surface receptors, which bind a range of ligands, including modified low-density lipoproteins and nucleic acids (4). SR-As are characterized by the presence of a collagen-like domain and include macrophage scavenger receptor type A (SR-A; SCARA1) (5), MARCO (SCARA2) (6), CSR1 (SCARA3) (7), SRCL4 (SCARA4) (8) and SCARA5 (9). These receptors are known to make important contributions to host defense. For example, Suzuki et al showed that SR-A $\%$ mice were more susceptible to Listeria monocytogenes infection, compared with wild-type control mice (10). Several previous studies have demonstrated that SCARA5 is involved in cancer progression. For example, one study showed that inhibiting the downregulation of SCARA5 significantly attenuates the epithelial-to-mesenchymal transition-associated migration of human lung carcinoma A549 cells that is induced by transforming growth factor- $\beta 1$ (11). Another study reported that SCARA5 knockdown markedly enhanced human hepatocellular carcinoma (HCC) cell growth in vitro, colony formation in soft agar, and invasiveness, tumorigenicity, and lung metastasis in vivo (12). However, its role in the progression and metastasis of OS remains to be fully elucidated.

Therefore, the present study investigated whether SCARA5 is involved in OS tumor growth and metastasis, and examined the potential underlying mechanisms as SCARA5 may be a potential therapeutic target for the treatment of patients with OS.

\section{Materials and methods}

Tissue specimens. Fresh OS tissue specimens were collected from 26 patients who underwent surgery for OS resection at Dongfang Hospital Affiliated to Beijing University of 
Chinese Medicine (Beijing, China) between October 2012 and May 2014. The group of patients with OS comprised 14 males and 12 females, aged between 13 and 58 years. Without any preoperative treatment, all 26 cases were pathologically diagnosed as OS postoperatively. In addition, 22 normal bone tissue specimens were collected from the long bones of 22 healthy subjects aged 24-61 years (males, 8; females, 14). The specimens were immediately preserved in liquid nitrogen (Leshan Dongya Cryogenic Vessel Co., Ltd, Beijing, China) for subsequent analyses. All subjects provided written informed consent, and the specimen collection procedure of the present study was approved by the Medical Ethics Committee of Dongfang Hospital Affiliated to Beijing University of Chinese Medicine (Beijing, China).

Cell culture. The U2OS and MG63 human OS cell lines and normal osteoblast cell line (hFOB1.19) were purchased from the American Type Culture Collection (Manassas, VA, USA). hFOB1.19 normal osteoblast cell line was used as the control group when evaluating the expression levels of SCARA5 in OS cells. All OS cell lines were cultured in complete Dulbecco's modified Eagle's medium (DMEM; Invitrogen; Thermo Fisher Scientific, Inc., Waltham, MA, USA), supplemented with $10 \%$ fetal bovine serum (Invitrogen; Thermo Fisher Scientific, Inc.), $1 \%$ L-glutamine, and $1 \%$ penicillin/streptomycin (both Sigma-Aldrich, St. Louis, MO, USA). The cultures were incubated at $37^{\circ} \mathrm{C}$ with $5 \% \mathrm{CO}_{2}$ in a humidified incubator.

Reverse transcription-quantitative polymerase chain traction (RT-qPCR) analysis. OS tissues were frozen in liquid nitrogen, washed twice with phosphate-buffered saline and lysed using ice-cold radioimmunoprecipitation assay buffer and 0.1 PMSF supplemented with a protease inhibitor cocktail (Sigma-Aldrich). Following centrifugation at $12,000 \mathrm{x} \mathrm{g}$ for $5 \mathrm{~min}$, the supernatant was collected. When the cells reached 90\% confluency, total RNA was extracted from the OS tissues and cells using TRIzol reagent, according to the manufacturer's protocol (Invitrogen; Thermo Fisher Scientific, Inc.). Subsequently, $2 \mu \mathrm{g}$ of total RNA was reverse transcribed to first-strand cDNA using TaqMan reverse transcription reagents (Applied Biosystems; Thermo Fisher Scientific, Inc.). The following primers were used: SCARA5, sense 5'-CAGCTG GTTTCTTACCACGTAT-3' and antisense 5'-GCACAAGTT CTCCCACACTTAG-3'; and $\beta$-actin, sense 5'-CCGTGAAAA GATGACCCAGATC-3' and antisense 5'-CACAGCCTGGAT GGCTACGT-3'. RT-qPCR was performed using $1 \mu 1 \mathrm{cDNA}$ templates, $2 \mu \mathrm{l}$ forward and reverse primers and $5 \mu \mathrm{l}$ SYBR Green qPCR Master Mix. Thermal cycling was performed as follows: $95^{\circ} \mathrm{C}$ for $10 \mathrm{~min}$, then 40 cycles of $95^{\circ} \mathrm{C}$ for $15 \mathrm{sec}$, $59^{\circ} \mathrm{C}$ for $30 \mathrm{sec}, 72^{\circ} \mathrm{C}$ for $30 \mathrm{sec}$, followed by an extra extension at $72^{\circ} \mathrm{C}$ for $5 \mathrm{~min}$. Reactions were performed using a Step One Plus Real-Time PCR machine (Applied Biosystems; Thermo Fisher Scientific, Inc.). For analysis, the expression levels of the target gene were normalized by the gene expression of $\beta$-actin. Based on the $\Delta \Delta \mathrm{Cq}$ method (13), the relative quantities of mRNA were expressed as $2^{-\Delta \Delta C q}$.

Western blot analysis. Proteins were extracted from the OS tissues and cells, and protein concentrations were measured using the Bradford method (14). Subsequently, $30 \mu \mathrm{g}$ of protein was separated by $10 \%$ SDS-PAGE and transferred onto a polyvinylidene fluoride membrane (both Sigma-Aldrich). The membrane was incubated with $2 \%$ nonfat dry milk in Tris-buffered saline (TBS; Sigma-Aldrich), to block non-specific binding, at room temperature for $1 \mathrm{~h}$. Subsequently, the membrane was immunoblotted with the following mouse monoclonal primary antibodies: Anti-SCARA5 (1:1,500; sc-98123), anti-phosphorylated (p)-FAK (Y397; sc-11765), anti-FAK (both 1:1,000; sc-271195), anti-p-Src (Y416; sc-101802), anti-Src (both 1:2,000; sc-24621), anti-matrix metalloproteinase 2 (MMP2; sc-13594), anti-MMP9 (sc-21733) and anti- $\beta$-actin (all 1:1,000; sc-8432; all Santa Cruz Biotechnology, Inc., Santa Cruz, CA, USA) overnight at $4^{\circ} \mathrm{C}$, followed by three washes in TBS with $0.05 \%$ Tween- 20 (Sigma-Aldrich) for $10 \mathrm{~min}$. Subsequently, the membranes were incubated with rat anti-mouse horseradish peroxidase-conjugated secondary antibody (1:3,000; sc-2370; Santa Cruz Biotechnology, Inc.) for $1 \mathrm{~h}$ at room temperature. The expression of the target protein was visualized using enhanced chemiluminescence (Pierce Biotechnology; Thermo Fisher Scientific, Inc.). Absorbance values of the target proteins were analyzed using Image-Pro Plus 6.0 (Media Cybernetics, Inc., Rockville, MD, USA).

SCARA5 expression vector construction and transfection. To construct the SCARA5 recombinant adenovirus vector, the cDNA encoding SCARA5 was amplified and subcloned into the adenoviral shuttle vector, pAd-CMV, and green fluorescent protein (GFP; both Invitrogen; Thermo Fisher Scientific, Inc.) was used as a non-specific control. The pAd-CMV adenoviral shuttle vector and pAdEasy-1 (Invitrogen; Thermo Fisher Scientific, Inc.) adenoviral gene expression vector were homologously recombined in the Escherichia coli strain, BJ5183 (Type Culture Collection of the Chinese Academy of Sciences, Shanghai, China) at $4^{\circ} \mathrm{C}$ for $16 \mathrm{~h}$. The newly recombined plasmid, Ad-SCARA5, was then propagated in 293 cells (Type Culture Collection of the Chinese Academy of Sciences) at $37^{\circ} \mathrm{C}$ for $16 \mathrm{~h}$. The recombinant adenoviruses were harvested, and the titers were determined using a p24 ELISA kit (Cell Biolabs, Inc., San Diego, CA, USA) prior to use.

For in vitro transfection, the OS cells $\left(3 \times 10^{3}\right)$ were seeded in 96-well plates. The cells grown to $30-50 \%$ confluence and were transfected with Ad-SCARA5 at final concentrations of $50 \mathrm{nM}$, using Lipofectamine 2000 transfection reagent (Invitrogen; Thermo Fisher Scientific, Inc.), according to the manufacturer's protocol.

Cell proliferation assay. To analyze cell proliferation, an MTT assay was performed. The transiently transfected cells were seeded in a 96-well plate at a cell density of $2 \times 10^{3}$ and were cultured at $24 \mathrm{~h}$ intervals for 4 days at room temperature. Subsequently, the initial culture medium was replaced with fresh medium containing MTT $(5 \mathrm{mg} / \mathrm{ml}$; Sigma-Aldrich, St. Louis, MO, USA) and incubated for an additional $4 \mathrm{~h}$ at room temperature. The formazan was dissolved in dimethylsulfoxide (150 $\mu \mathrm{l} /$ well; Sigma-Aldrich) for $10 \mathrm{~min}$. The absorbance was measured at $570 \mathrm{~nm}$ using a Spectra Max 190 microplate reader (Molecular Devices, LLC, Sunnyvale, CA, USA). All experiments were independently repeated at least three times. 
A

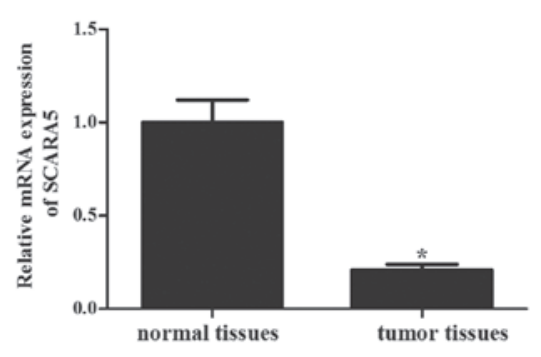

C

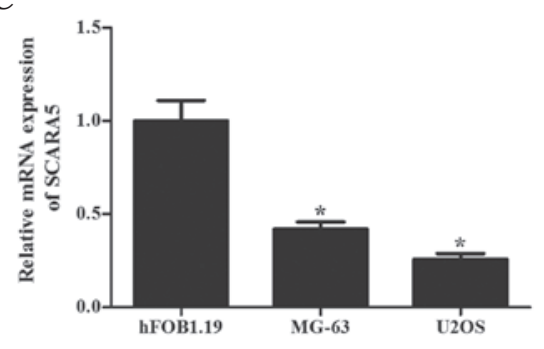

B
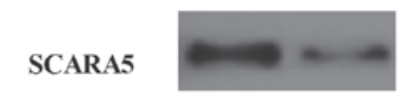

$\beta$-actin

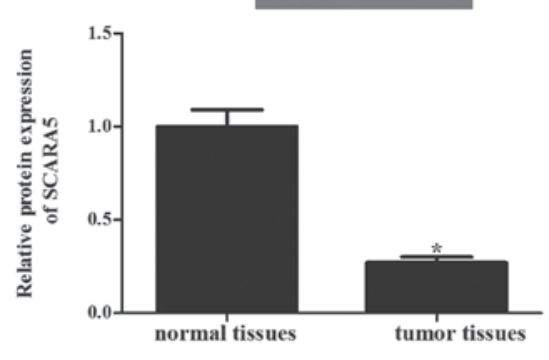

D SCARA5
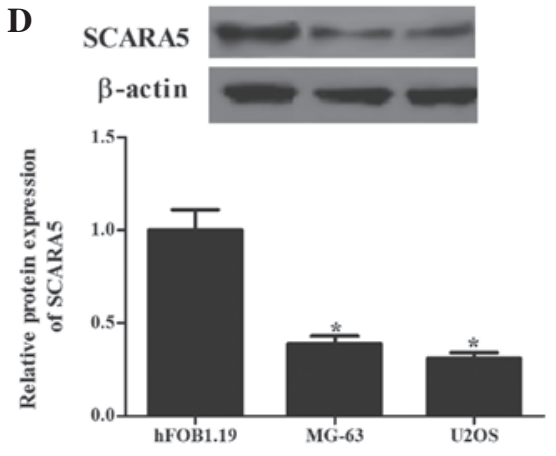

Figure 1. SCARA5 is expressed at low levels in OS tissues and cell lines. (A) mRNA expression levels of SCARA5 were analyzed busing reverse transcription-quantitative polymerase chain reaction analysis. The mRNA levels of SCARA5 in the OS tissues were significantly lower, compared with those in the normal bone tissues. (B) Representative western blot images of the SCARA5 protein in OS tissues. (C) Representative mRNA expression levels of SCARA5 in OS cell lines. (D) Representative western blot images of the SCARA5 protein in OS cell lines. Data are expressed as the mean \pm standard deviation from experiments performed in triplicate. ${ }^{*} \mathrm{P}<0.05$, vs. normal tissues/hFOB1.19 cells. OS, osteosarcoma; SCARA5, scavenger receptor class A, member 5.

Colony formation assay. For the soft agar colony formation assay, $2 \times 10^{4}$ cells were plated in 24-well plates and grown on a plate containing $1 \%$ base agar (Sigma-Aldrich) and $0.5 \%$ top agar for 21 days at room temperature, until colonies formed. The colonies were stained with $1 \%$ crystal violet for $30 \mathrm{sec}$ following fixation with $10 \%$ formaldehyde (both Sigma-Aldrich) for $5 \mathrm{~min}$. The numbers of colonies were counted under a dissecting microscope (BIX-103G, Luxi Chemical Group Co., Ltd., Beijing, China). All experiments were independently repeated at least three times.

Cell migration and invasion assays. OS cells ( $1 \times 10^{4}$ cells/well) in $200 \mu 1$ serum-free DMEM were added to the upper chamber of a Transwell (Invitrogen; Thermo Fisher Scientific, Inc.) with an $8 \mu \mathrm{m}$ microporous filter (Sigma-Aldrich), following which $500 \mu \mathrm{l}$ DMEM containing $10 \%$ FBS was added to the lower chamber. Following $24 \mathrm{~h}$ incubation at $37^{\circ} \mathrm{C}$, the cells on the lower surface of the filter were fixed in methanol, stained with Giemsa (Sigma-Aldrich) and examined under a Leica DM5000B microscope (Leica Microsystems GmbH, Wetzlar, Germany). The average numbers of migrated cells from five randomly-selected optical fields and triplicate filters were determined.

For in vitro invasion assays, the cells were suspended in a volume of $50 \mu \mathrm{l}$ serum-free medium, which was then added to the upper chamber of a chemotaxis chamber (Neuro Probe, Inc., Gaithersburg, MD, USA). Complete medium was added to the lower chamber. A polycarbonate membrane (Sigma-Aldrich) was placed between the two chambers, and culture medium supplemented with $20 \mu \mathrm{l}$ Matrigel (BD Biosciences, Franklin Lakes, NJ, USA) was applied. After $24 \mathrm{~h}$ at room temperature, the non-invading cell remaining on the upper surface were removed using cotton-tipped swabs, following which the filters were fixed in methanol for $3 \mathrm{~min}$ and stained with $0.05 \%$ crystal violet in phosphate-buffered saline for $15 \mathrm{~min}$. The cells on the underside of the filters were visualized and counted under a Leica DM5000B microscope (Leica Microsystems GmbH). Each sample was assayed in triplicate.

Statistical analysis. All experiments were performed independently, at least three times. Differences between groups were analyzed via Student's t-test using SPSS 13.0 software (IBM Corp., Armonk, NY, USA). Data are expressed as the mean \pm standard deviation from three independent experiments performed in triplicate. $\mathrm{P}<0.05$ was considered to indicate a statistically significant difference.

\section{Results}

Expression of SCARA5 at low levels in OS tissues and cell lines. The present study first determined the mRNA and protein levels of SCARA5 in human OS tissues. As shown in Fig. 1, the mRNA (Fig. 1A) and protein (Fig. 1B) expression levels of SCARA5 in the OS tissues were significantly lower, compared to those in the normal bone tissues $(\mathrm{P}<0.05)$. The expression levels of SCARA5 in human OS cells (MG-63 and 
A

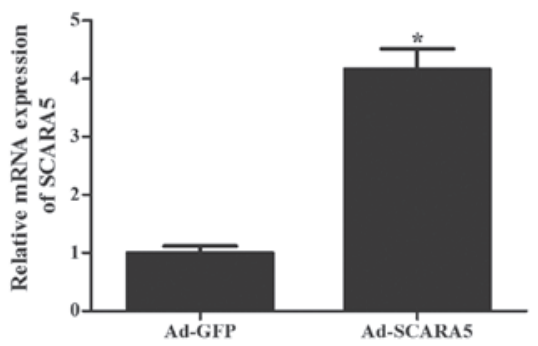

B

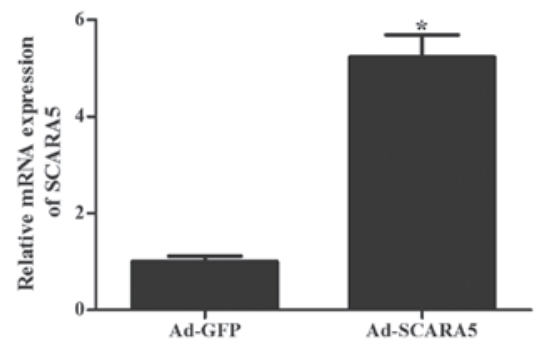

MG63

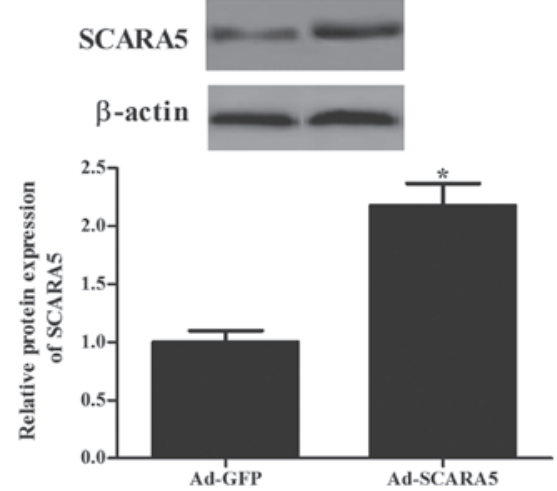

U2OS

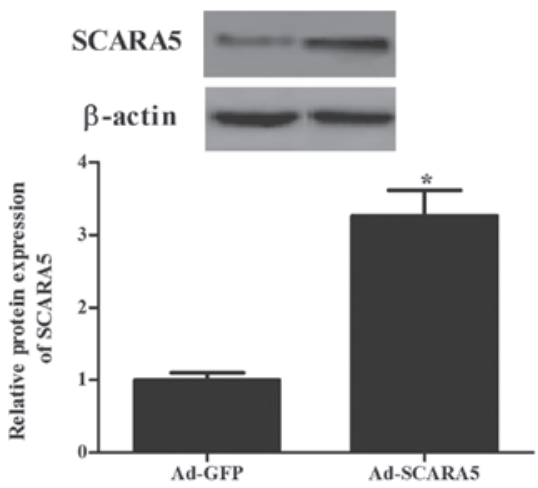

Figure 2. Expression levels of SCARA5 in MG63 and U2OS cell lines. The cells were transfected with Ad-GFP and Ad-SCARA5, respectively. The corresponding transfection efficiency was detected using reverse transcription-quantitative polymerase chain reaction and Western blot analyses. (A) mRNA and protein expression levels of SCARA5 in MG63 cells. (B) mRNA and protein expression levels of SCARA5 in U2OS cells. The expression levels of SCARA5 were significantly upregulated in the Ad-SCARA5-transfected MG63 and U2OS cells, compared with the Ad-GFP-transfected cells. Data are expressed as the mean \pm standard deviation from experiments performed in triplicate. "P<0.05, vs. Ad-GFP group. SCARA5, scavenger receptor class A, member 5; GFP, green fluorescent protein.

A

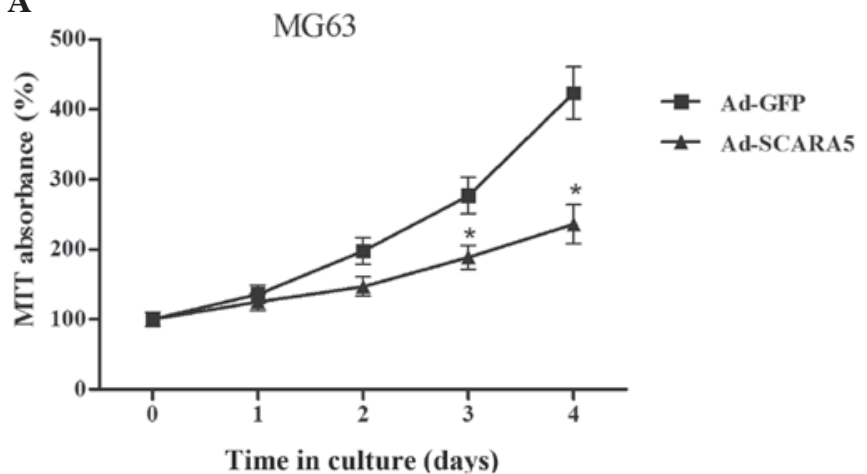

B

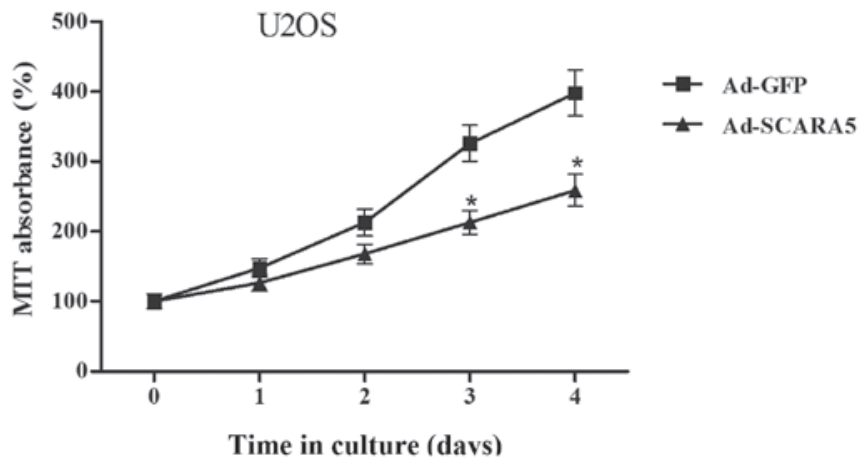

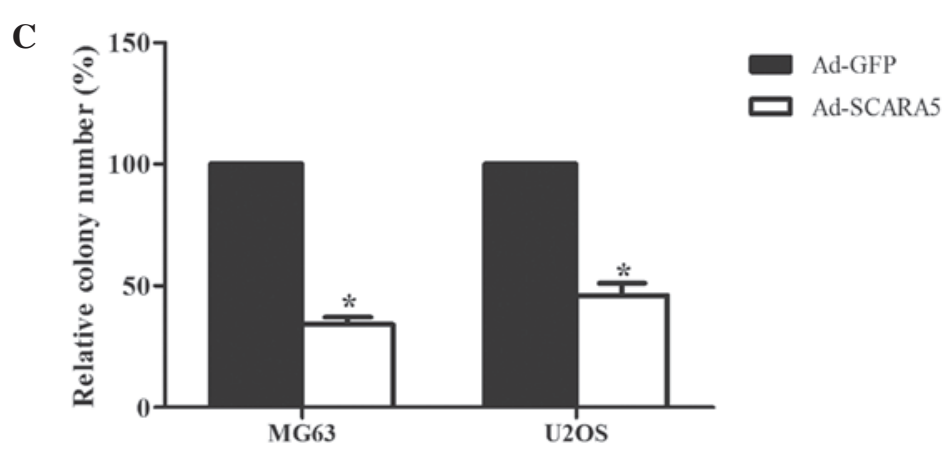

Figure 3. Overexpression of SCARA5 suppresses growth and colony formation of OS cells in vitro. (A and B) Effects of the overexpression of SCARA5 on MG63 and U2OS OS cell proliferation, were measured using an MTT assay. The growth of OS cells overexpressing SCARA5 was suppressed, compared with the Ad-GFP-transfected control cells. (C) Results of the colony formation assay showed a significant reduction in the numbers of colonies in the SCARA5-overexpressing MG63 and U2OS cells, compared with the control cells. Data are expressed as the mean \pm standard deviation from experiments performed in triplicate. ${ }^{*} \mathrm{P}<0.05$, vs. Ad-GFP group. OS, osteosarcoma; SCARA5, scavenger receptor class A, member 5; GFP, green fluorescent protein. 
A

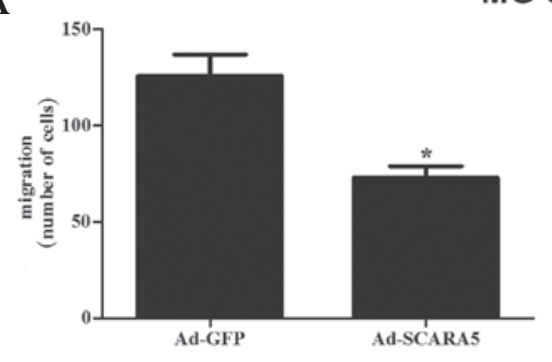

B

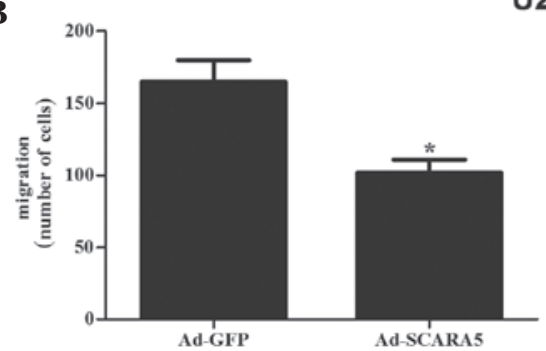

MG-63

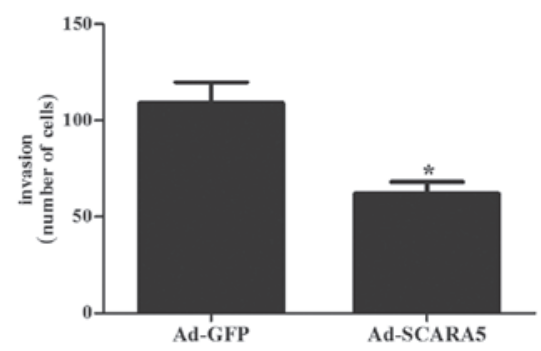

J2OS

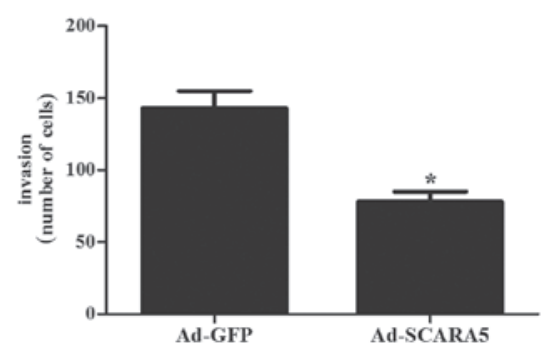

Figure 4. Overexpression of SCARA5 decreases osteosarcoma cell migration and invasion. A Transwell assay revealed that SCARA5 decreased migration in the MG63 (A, left) and U2OS (B, left) cells, compared with the Ad-GFP control cells. A Matrigel invasion assay revealed that SCARA5 decreased invasion in the MG63 (A, right) and U2OS (B, right) cells, compared with the Ad-GFP control cells. Data are expressed as the mean \pm standard deviation from experiments performed in triplicate. ${ }^{*} \mathrm{P}<0.05$, vs. Ad-GFP group. SCARA5, scavenger receptor class A, member 5; GFP, green fluorescent protein.

A

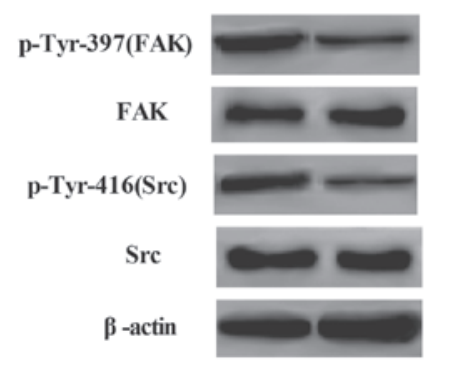

$\mathbf{B}$

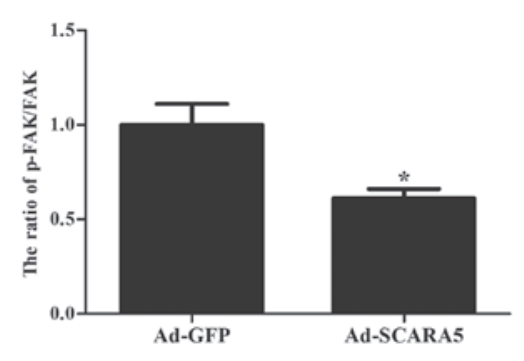

C

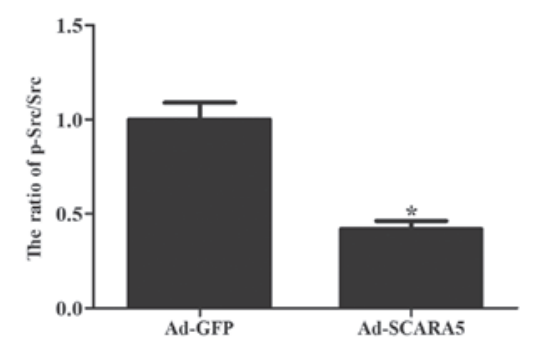

Figure 5. Overexpression of SCARA5 inhibits the phosphorylation of FAK (Tyr-397) and Src (Tyr-416) in MG63 cells. (A) Western blot analysis detected the protein expression levels of p-Tyr-397 and p-Tyr-416 in the treated groups with corresponding antibodies. (B and C) Relative protein expression levels of p-FAK and p-Src, quantified using Image-Pro Plus 6.0 and normalized to $\beta$-actin. Data are expressed as the mean \pm standard deviation from experiments performed in triplicate. " $\mathrm{P}<0.05$, vs. Ad-GFP group. SCARA5, scavenger receptor class A, member 5; p-, phosphorylated; FAK, focal adhesion kinase; GFP, green fluorescent protein.
A

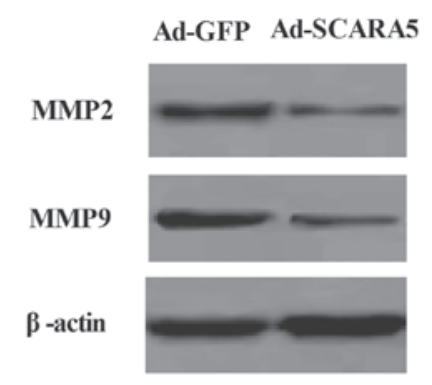

B

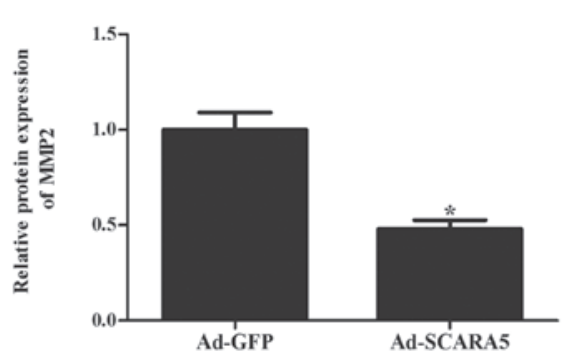

C

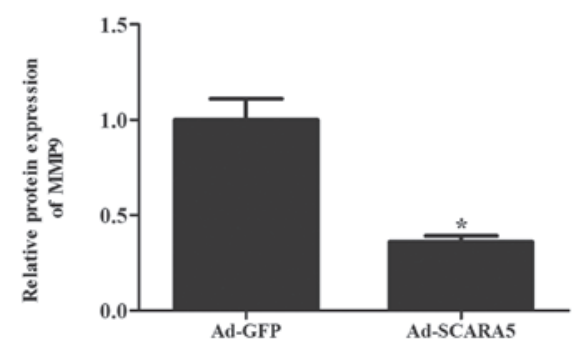

Figure 6. Overexpression of SCARA5 inhibits the expression levels of MMP2 and MMP9 in MG63 cells. (A) Western blot analysis was used to detect the the protein levels of MMP2 and MMP9 in the differently treated cell groups with the indicated antibodies. (B and C) Relative protein expression levels of MMP2 and MMP9 were quantified using Image-Pro Plus 6.0 software and normalized to $\beta$-actin. Data are expressed as the mean \pm standard deviation from experiments performed in triplicate. ${ }^{*} \mathrm{P}<0.05$, vs. Ad-GFP group. SCARA5, scavenger receptor class A, member 5; GFP, green fluorescent protein; MMP, matrix metalloproteinase. 
U2OS) were also analyzed. Consistent with the observation from the tissue samples, the expression levels of SCARA5 were significantly decreased in the two cell lines, compared with those in the normal human primary osteoblasts (Fig. 1C and D). These results indicated that SCARA5 may function as a tumor suppressor in OS.

Overexpression of SCARA5 inhibits cell proliferation and colony formation. To examine the biological significance of SCARA5 in OS tumorigenesis, the present study generated SCARA5-overexpressing MG-63 and U2OS OS cell lines. The transfection efficiency was confirmed using RT-qPCR and western blot analyses. Following SCARA5 transfection, the mRNA and protein levels of SCARA5 were significantly increased in the MG-63 (Fig. 2A) and U2OS (Fig. 2B) cells, respectively. The present study also examined the effect of SCARA5 overexpression on cell proliferation and colony formation. As shown in Fig. 3, transfection of the cells with Ad-SCARA5 significantly inhibited the growth of the MG-63 cells (Fig. 3A). It also significantly suppressed colony formation in the MG-63 cells (Fig. 3C). Similarly, the overexpression of SCARA5 suppressed growth (Fig. 3B) and colony formation (Fig. 3C) in the U2OS cells.

Overexpression of SCARA5 inhibits cell migration and invasion. The present study used Transwell assays to assess the effects of the expression of SCARA5 on cell migration and invasion. The results showed that cell migration was significantly inhibited by SCARA5 overexpression in the MG-63 (Fig. 4A, left) and U2OS cells (Fig. 4B, left), respectively. In addition, compared with the cells transfected with the empty vector, SCARA5 overexpression significantly inhibited the invasion of the MG-63 (Fig. 4A, right) and U2OS cells (Fig. 4B, right).

SCARA5 regulates activation of the FAK signaling pathway by affecting tyrosine phosphorylation. The FAK signaling pathway is important in cancer cell growth and invasion (15). To examine the molecular mechanisms by which SCARA5 contributes to these malignant features, the present study examined the effect of SCARA5 on the levels of tyrosine phosphorylation at specific sites of certain molecules involved in the FAK signaling pathway. The results of the Western blot analysis revealed that the overexpression of SCARA5 significantly inhibited the phosphorylation of the FAK residue (Tyr-397; Fig. 5A and B) and Src residue (Tyr-416; Fig. 5A and C) in the MG63 cells.

The present study also assessed the expression levels of MMP-2 and MMP-9, which are crucial downstream molecules in the FAK signaling pathway (16). As shown in Fig. 6, the overexpression of SCARA5 significantly inhibited the expression levels of MMP-2 (Fig.6A and B) and MMP-9 (Fig. 6A and C) in the MG63 cells, compared with the cells in the Ad-GFP group.

\section{Discussion}

In the present study, SCARA5 was found to be expressed at low levels in human OS tissues and cell lines, and provided the first evidence, to the best of our knowledge, that SCARA5 overexpression significantly inhibits the proliferation and reduces the metastatic activity of OS. Furthermore, the overexpression of SCARA5 inhibited the phosphorylation of FAK and downstream molecules in human OS cells.

SCARA3 was initially identified as a macrophage scavenger receptor homolog (17). A previous study showed that the expression of SCARA3 is downregulated in prostate cancer, and the downregulation of the expression of SCARA3 in prostate cancer cell lines is caused by methylation of its promoter (18). In the present study, SCARA5 was found to be expressed at low levels in OS tissues and cell lines, suggesting that SCARA5 may be a novel candidate tumor suppressor gene, which is downregulated in OS as a result of promoter hyper-methylation.

On obtaining the above results, the present study subsequently investigated the role of SCARA5 in OS cell growth. It was found that the overexpression of SCARA5 markedly reduced cell proliferation and colony formation, suggesting that SCARA5 was involved in abnormal cell proliferation in OS cells.

The ability of tumor cells to migrate and invade is considered an important indicator of cell aggressiveness and metastatic ability. Therefore, reducing cell migration and/or invasion is essential to inhibit tumor progression (19). A previous report showed that the overexpression of SCARA5 markedly enhanced HCC cell invasion in vitro and tumor metastasis in vivo (12). In the present study, the overexpression of SCARA5 was found to significantly inhibit the migration and invasion abilities of MG63 cells. These data indicated that SCARA5 is crucial in OS metastasis.

FAK, a non-receptor tyrosine kinase, localizes at focal adhesions, and is important in relaying extracellular signals between integrins and intracellular compartments (20). FAK is activated in a range of tumor cells, and the activated FAK forms a complex with Src and p130Cas, which leads to tumor growth and metastasis by promoting cell survival, cell cycle progression, motility and invasion $(21,22)$. The concept of targeting FAK as a therapeutic strategy for cancer treatment is promising (20). A previous report study by Seong et al demonstrated that short hairpin RNA-mediated knockdown of SATB2 decreases the migration and invasion of OS cells by suppressing the phosphorylation of FAK (23). Hu et al (24) showed that the overexpression of phosphatase and tensin homolog also inhibits migration and invasion through downregulating the expression of p-FAK. The silencing of galectin-3 also represses OS cell migration and invasion through inhibition of FAK/Src/Lyn activation (25). Notably, Huang et al also verified that SCARA5 inhibits HCC progression via the FAK signaling pathway (12). Similarly, in the present study, it was demonstrated that the overexpression of SCARA $5 n$ in MG-63 cells markedly inhibited the phosphorylation of FAK.

MMPs are important roles in the matrix degradation required for tumor growth and invasion (26). It has been reported that MMP-9 is directly associated with metastatic processes in OS $(27,28)$. MMP2 is also a prominent member of the MMP family, and patients with OS exhibiting high expression levels of MMP2 have a poor prognosis (29). In addition, MMP-2 and MMP-9 are crucial downstream molecules in the FAK signaling pathway. In the present study, the overexpression of SCARA5 was observed to significantly inhibit 
the expression levels of MMP-2 and MMP-9 in MG63 cells, compared with cells in the Ad-GFP-transfected group. These results suggested that the overexpression of SCARA5 may contribute to the downregulation of p-FAK and downstream molecules in MG63 cells, which may lead to reduced tumor cell growth and invasion.

In conclusion, the present study showed that SCARA5 may be important in tumor growth and metastasis, and that SCARA5 may be a potential therapeutic target for the treatment of OS.

\section{References}

1. Mirabello L, Troisi RJ and Savage SA: Osteosarcoma incidence and survival rates from 1973 to 2004. Cancer 115: 1531-1543, 2009.

2. Amankwah EK, Conley AP and Reed DR: Epidemiology and therapies for metastatic sarcoma. Clin Epidemiol 5: 147-162, 2013.

3. Friedman MA and Carter SK: The therapy of osteogenic sarcoma: Current status and thoughts for the future. J Surg Oncol 4: 482-510, 1972

4. Peiser L and Gordon S: The function of scavenger receptorsexpressed by macrophages and their role in the regulation of inflammation. Microbes Infect 3: 149-159, 2001.

5. Platt N, Haworth R, Darley L and Gordon S: The many roles of the class A macrophage scavenger receptor. Int Rev Cytol 212: $1-40,2002$.

6. Elomaa O, Sankala M, Pikkarainen T, Bergmann U, Tuuttila A, Raatikainen-Ahokas A, Sariola $\mathrm{H}$ and Tryggvason K: Structure of the human macrophage MARCO receptor and characterization of its bacteria-binding region. J Biol Chem 273: 4530-4538, 1998.

7. Han HJ, Tokino $T$ and Nakamura Y: CSR, a scavenger receptor-like protein with a protective role against cellular damage caused by UV irradiation and oxidative stress. Hum Mol Genet 7: 1039-1046, 1998.

8. Ohtani K, Suzuki Y, Eda S, Kawai T, Kase T, Keshi H, Sakai Y, Fukuoh A, Sakamoto T, Itabe H, et al: The membrane-type collectin CL-P1 is a scavenger receptor on vascular endothelial cells. J Biol Chem 276: 44222-44228, 2001.

9. Jiang Y, Oliver P, Davies KE and Platt N: Identification and characterization of murine SCARA5, a novel class A scavenger receptor that is expressed by populations of epithelial cells. J Biol Chem 281: 11834-11845, 2006

10. Suzuki H, Kurihara Y, Takeya M, Kamada N, Kataoka M, Jishage K, Ueda O, Sakaguchi H, Higashi T, Suzuki T, et al: A role for macrophage scavenger receptors in atherosclerosis and susceptibility to infection. Nature 386:292-296, 1997.

11. Liu J, Hu G, Chen D, Gong AY, Soori GS, Dobleman TJ and Chen XM: Suppression of SCARA5 by Snaill is essential for EMT-associated cell migration of A549 cells. Oncogenesis 2: e73, 2013

12. Huang J, Zheng DL, Qin FS, Cheng N, Chen H, Wan BB, Wang YP, Xiao HS and Han ZG: Genetic and epigenetic silencing of SCARA5 may contribute to human hepatocellular carcinoma by activating FAK signaling. J Clin Invest 120: 223-241, 2010.
13. Livak KJ and Schmittgen TD: Analysis of relative gene expression data using real-time quantitative PCR and the $2^{-\Delta \Delta \mathrm{C}}$ method. Methods 25: 402-408, 2001.

14. Kruger NJ: The Bradford method for protein quantitation. Methods Mol Biol 32: 9-15, 1994.

15. Sulzmaier FJ, Jean C and Schlaepfer DD: FAK in cancer: Mechanistic findings and clinical applications. Nat Rev Cancer 14: 598-610, 2014.

16. Fingleton B: Matrix metalloproteinases: Roles in cancer and metastasis. Front Biosci 11: 479-491, 2006.

17. Yu G, Tseng GC, Yu YP, Gavel T, Nelson J, Wells A, Michalopoulos G, Kokkinakis D and Luo JH: CSR1 suppresses tumor growth and metastasis of prostate cancer. Am J Pathol 168: 597-607, 2006

18. Kurayoshi M, Oue N, Yamamoto H, Kishida M, Inoue A, Asahara T, Yasui W and Kikuchi A: Expression of Wnt-5a is correlated with aggressiveness of gastric cancer by stimulating cell migration and invasion. Cancer Res 66: 10439-10448, 2006.

19. Bock AJ, Nymoen DA, Brenne K, Kærn J and Davidson B: SCARA3 mRNA is overexpressed in ovarian carcinoma compared with breast carcinoma effusions. Hum Pathol 43: 669-674, 2012.

20. Sieg DJ, Hauck CR, Ilic D, Klingbeil CK, Schaefer E, Damsky CH and Schlaepfer DD: FAK integrates growth-factor and integrin signals to promote cell migration. Nat Cell Biol 2: 249-256, 2000.

21. Tilghman RW and Parsons JT: Focal adhesion kinase as a regulator of cell tension in the progression of cancer. Semin Cancer Biol 18: 45-52, 2008.

22. Schlaepfer DD, Mitra SK and Ilic D: Control of motile and invasive cell phenotypes by focal adhesion kinase. Biochim Biophys Acta 1692: 77-102, 2004.

23. Seong BK, Lau J, Adderley T, Kee L, Chaukos D, Pienkowska M, Malkin D, Thorner P and Irwin MS: SATB2 enhances migration and invasion in osteosarcoma by regulating genes involved in cytoskeletal organization. Oncogene 34: 3582-3592, 2015.

24. Hu Y, Xu S, Jin W, Yi Q and Wei W: Effect of the PTEN gene on adhesion, invasion and metastasis of osteosarcoma cells. Oncol Rep 32: 1741-1747, 2014.

25. Park GB, Kim DJ, Kim YS, Lee HK, Kim CW and Hur DY: Silencing of galectin-3 represses osteosarcoma cell migration and invasion through inhibition of FAK/Src/Lyn activation and $\beta$-catenin expression and increases susceptibility to chemotherapeutic agents. Int J Oncol 46: 185-194, 2015.

26. Chambers AF and Matrisian LM: Changing views of the role of matrix metalloproteinases in metastasis. J Natl Cancer Inst 89: 1260-1270, 1997.

27. Kido A, Tsutsumi M, Iki K, Takahama M, Tsujiuchi T, Morishita T, Tamai S and Konishi Y: Overexpression of matrix metalloproteinase (MMP)-9 correlates with metastatic potency of spontaneous and 4-hydroxyaminoquinoline 1-oxide (4-HAQO)-induced transplantable osteosarcomas in rats. Cancer lett 137: 209-216, 1999.

28. Himelstein BP, Asada N, Carlton MR and Collins $\mathrm{MH}$ : Matrix metalloproteinase.9 (MMP.9) expression in childhood osseous osteosarcoma. Med Pediatr Oncol 31: 471-474, 1998.

29. Uchibori M, Nishida Y, Nagasaka T, Yamada Y, Nakanishi K and Ishiguro N: Increased expression of membrane-type matrix metalloproteinase-1 is correlated with poor prognosis in patients with osteosarcoma. Int J Oncol 28: 33-42, 2006. 